\title{
Influences on the radius of the auroral oval
}

\author{
S. E. Milan ${ }^{1}$, J. Hutchinson ${ }^{1}$, P. D. Boakes ${ }^{1,2}$, and B. Hubert ${ }^{3}$ \\ ${ }^{1}$ Department of Physics and Astronomy, University of Leicester, Leicester LE1 7RH, UK \\ ${ }^{2}$ British Antarctic Survey, Natural Environment Research Council, High Cross, Cambridge, UK \\ ${ }^{3}$ Laboratory of Planetary and Atmospheric Physics, University of Liége, Liége, 4000 Belgium
}

Received: 2 March 2009 - Revised: 11 June 2009 - Accepted: 20 July 2009 - Published: 23 July 2009

\begin{abstract}
We examine the variation in the radius of the auroral oval, as measured from auroral images gathered by the Imager for Magnetopause-to-Aurora Global Exploration (IMAGE) spacecraft, in response to solar wind inputs measured by the Advanced Composition Explorer (ACE) spacecraft for the two year interval June 2000 to May 2002. Our main finding is that the oval radius increases when the ring current, as measured by the Sym-H index, is intensified during geomagnetic storms. We discuss our findings within the context of the expanding/contracting polar cap paradigm, in terms of a modification of substorm onset conditions by the magnetic perturbation associated with the ring current.
\end{abstract}

Keywords. Magnetospheric physics (Magnetospheric configuration and dynamics; Solar wind-magnetosphere interactions; Storms and substorms)

\section{Introduction}

Contractions and expansions of the Earth's auroral ovals, like geomagnetic activity, are governed by the conditions within the solar wind. As early as the Carrington Storm of 1859 a link between extreme solar activity and the appearance of aurora at unusually low latitudes was established (e.g., Cliver, 2006). Subsequently, Feldstein and Starkov (1967) showed that the changing latitudinal location and extent of the auroral oval could be parameterized by the $Q$ index of geomagnetic activity, and several authors demonstrated a link between the location of the oval and the $D_{s t}$ index (e.g., Akasofu and Chapman, 1963; Meng, 1982, 1984; Yokoyama et al., 1998). The interplanetary magnetic field (IMF), and especially its north-south component IMF $B_{Z}$, is also known to control the location of the aurora (e.g., Holzworth and Meng,

\section{Correspondence to: S. E. Milan}

(steve.milan@ion.le.ac.uk)
1975; Hardy et al., 1981; Nakai et al., 1986), though as $D_{s t}$ is itself related to IMF $B_{Z}$ (e.g., Russell et al., 1974; Burton et al., 1975) there is still some debate over which ultimately controls the oval radius. Moreover, significant variations in the location of the aurora take place during the substorm cycle, caused by the waxing and waning of open magnetic flux in the magnetosphere driven by magnetic reconnection at the dayside magnetopause and in the magnetotail as part of the Dungey cycle of magnetospheric convection (e.g., Dungey, 1961; Siscoe and Huang, 1985; Craven and Frank, 1988; Cowley and Lockwood, 1992; Lockwood and Cowley, 1992; Milan et al., 2003, 2007, 2008). The present paper investigates the factors influencing the radius of the auroral oval, specifically the intensity of the ring current measured by the Sym-H index, the solar wind conditions, including solar wind speed and density, the orientation of the IMF, and the substorm cycle. We employ auroral observations from two years of operation of the Imager for Magnetopauseto-Aurora Global Exploration (IMAGE) spacecraft (Burch et al., 2000), constituting a statistical study of the radius of the auroral oval of unprecedented size.

The expanding/contracting polar cap paradigm (ECPC) provides a powerful conceptual framework within which to understand coupling between the solar wind and the magnetosphere, the excitation of ionospheric convection, and the role of substorms in the convection cycle. However, while the rate at which the polar cap grows in response to solar wind conditions has been investigated (e.g., Milan, 2004; Milan et al., 2007, 2008), the ECPC does not address the factors that determine how far the polar cap must expand before nightside reconnection is excited, that is before substorm expansion phase onset occurs, and the polar cap contracts. Indeed, it has been shown that the open magnetic flux content of the magnetosphere (polar cap size) at substorm onset is variable (e.g., Milan, 2004; Shukhtina et al., 2005; Milan et al., 2007, 2008; Boakes et al., 2009). In some cases this variability may be attributed to the triggering of substorms

Published by Copernicus Publications on behalf of the European Geosciences Union. 
by features in the solar wind, such as solar wind pressure steps (e.g., Boudouridis et al., 2003; Milan et al., 2004) or northward turnings of the IMF (e.g., Caan et al., 1977; Rostoker, 1983), which occur irrespective of the polar cap size. However, probably the majority of substorms occur spontaneously as the polar cap is expanding - that is, they display a growth phase prior to onset - and the size at which this occurs is variable (e.g., Milan et al., 2007, 2008; Boakes et al., 2009). Shukhtina et al. (2005) demonstrated a relationship between open flux at substorm onset and the integrated southward component of the interplanetary magnetic field (IMF $B_{Z}$ ) in the $30 \mathrm{~min}$ prior to onset. This is consistent with the accumulation of open flux during the growth phase, but this still does not prescribe the open flux level at which onset will occur.

One possible controlling factor is the intensity of the ring current. Milan et al. (2008) demonstrated a clear dependence of the latitude of substorm onset on the Sym-H index, a measure of the magnetic perturbation associated with the ring current, indicating that the auroral oval expands to low latitudes when the ring current is enhanced. Before this, Meng (1982, 1984) and Yokoyama et al. (1998), amongst others, showed a link between the latitude of the auroral oval and $D_{s t}$, another measure of ring current intensity. As suggested by Nakai and Kamide (2003), and reiterated by Milan et al. (2008), the enhanced ring current found during geomagnetic storms could alter the magnetic field geometry of the nearEarth magnetotail, delaying substorm onset until the open flux content of the magnetosphere grows unusually large. This would naturally explain the observation of aurora at low latitudes during periods of intense geomagnetic disturbance, particularly geomagnetic storms.

The ring current is enhanced during geomagnetic storms, which are usually initiated by the impact of a solar wind pressure step on the magnetosphere, triggering the injection of plasma from the magnetotail into the inner magnetosphere. Sym- $\mathrm{H}$ measures the perturbation of the north-south magnetic field component observed by a ring of magnetometers located around the Earth's magnetic equator. A positive perturbation, the initial phase, is observed as the magnetosphere is compressed by the high-pressure solar wind. This is followed by a rapid decrease in Sym-H to negative values as the enhanced ring current produces a southward perturbation in the field, the main phase. Values of Sym-H of -100 and $-300 \mathrm{nT}$ indicate moderate and strong storm conditions. There is then a gradual reduction of Sym-H to quiet-time values as the ring current plasma precipitates into the ionosphere over the next few days, the recovery phase. This paper investigates changes in the radius of the auroral oval as Sym-H progresses through this characteristic variation.

\section{Methodology}

In this study we use observations of the Northern Hemisphere UV aurora from the 2-year period June 2000 to May 2002 provided by the Far Ultraviolet (FUV) instrument (Mende et al., 2000a,b) onboard the IMAGE satellite. FUV comprised the Wideband Imaging Camera (WIC) and the Spectrographic Imager (SI). WIC was sensitive to auroral emissions over a broad range of the UV spectrum, the primary component of which is associated with precipitating electrons. The SI12 channel imaged Doppler-shifted Lyman$\alpha$ emission associated with precipitating protons. The orbital geometry and spin of the IMAGE spacecraft was such that images were captured by each camera with a cadence of 2 min for approximately $10 \mathrm{~h}$ of each 14 -h orbit. Due to contamination of the WIC observations by dayglow we discarded WIC images from the months May, June, and July. Our Northern Hemisphere dataset thus comprised close to 350000 images from SI12 and 260000 images from WIC.

Several methods are available for determining the open magnetic flux content of the magnetosphere from the size of the dim region enclosed by the auroral oval, the polar cap (e.g., Carbary et al., 2003; Hubert et al., 2006; Boakes et al., 2008). These methods identify the open/closed field line boundary (OCB) as the poleward edge of the luminosity associated with the main auroral oval. However, these methods tend to fail when dayglow encroaches on the auroral oval (especially a problem when the oval has expanded to low latitudes), when the auroral oval is dim, or where the latitudinal profile of auroral luminosity in any local time sector departs significantly from expectations. Here, we have developed a more robust method of determining the radius of the oval in each available image. This method is approximate, and does not provide a quantitative estimate of the open magnetic flux threading the polar cap. However, it greatly increases the proportion of images that can be included in our statistics.

We perform the fitting on SI12 images of the proton auroral oval as these are affected less by dayglow. Three examples are shown in the upper panels of Fig. 1, including a contracted oval, an oval of roughly typical size, and an expanded oval. Inspection of many SI12 images shows that the auroral oval can usually be approximated by a circle displaced antisunwards from the geomagnetic pole, as discussed previously by Holzworth and Meng (1975), Meng et al. (1977), and references therein. For the proton aurora we find that this antisunwards offset is approximately $5^{\circ}$ of latitude. There also tends to be a displacement duskwards by approximately $2^{\circ}$, as previously discussed by Boakes et al. (2008) and Milan et al. (2009). Circles centred on this point, $O$, with radii between $8^{\circ}$ and $35^{\circ}$, are tested. For each circle we determine the SI1 2 brightness at 200 points equally spaced around its circumference, from which we calculate the mean brightness $I$; points where image data is missing do not contribute to the mean. The lower panels of Fig. 1 show how $I$ varies as a function of radius for each image. The best-fit oval radius 
$\lambda$ is that at which $I$ maximizes. If fewer than 100 points contributed to the calculation of the mean, i.e. only a very partial image is available, the measurement is discarded. The best-fit oval is shown as a black and white dashed circle superimposed on each image. We use the maximum value of $I$, hereafter referred to as overall SI12 brightness or $I_{\text {oval }}$, as a general measure of the intensity of the auroral oval. Although rather crude, this method of deterimining the radius is surprisingly robust. As shown in the middle panel of Fig. 1, a reasonable fit is provided even in the presence of dayglow, in the case of only partial images, and for images in which instrumental artefacts are present (in this case in the bottom right hand corner of the panel).

As well as the overall brightness in each SI12 image, $I_{\text {oval }}$, we determine the maximum brightness on the nightside of each image, $I_{\mathrm{SI} 12}$, which we also call the peak SI12 brightness; the position of this peak is indicated in the three examples of Fig. 1, indicating that occasionally this can correspond to artefacts. In addition we determine the maximum nightside brightness in each corresponding WIC image, where WIC images are available, $I_{\text {WIC }}$; the overall brightness in WIC images is not used due to the contribution of dayglow. $I_{\text {oval }}$ represents the overall brightness of the oval, whereas $I_{\mathrm{SI} 12}$ and $I_{\mathrm{WIC}}$ indicate the brightness of nightside auroral features such as the substorm auroral bulge.

Figure 2 shows the occurrence distribution of oval radius $\lambda$, determined from over 308000 images. The overall distribution (solid curve) peaks at an oval radius of $19^{\circ}$, falling at higher and lower radii. As will be discussed below, at the extremes of the distribution, especially at the highest radii, some points are probably associated with bad fits to the auroral oval. These appear to occur most often when the auroral oval is extremely dim and the maximum in $I$ corresponds to the dayglow.

The inset panel of Fig. 2 shows a comparison between oval radius and the simultaneous open magnetic flux content of the polar cap, $F_{\mathrm{PC}}$, for a subset of the 2-year dataset. The method of Boakes et al. $(2008,2009)$ has been applied to those WIC images which were not contaminated by dayglow (i.e. images from dates near the winter solstices) to calculate $F_{\mathrm{PC}}$. This method identifies the poleward boundary of the auroral emission in all local time sectors, and assumes that this is a proxy for the open/closed field line boundary (OCB). $F_{\mathrm{PC}}$ is then calculated by integrating the magnetic flux through the polar cap, assuming that the field is dipolar. The occurrence distribution of Fig. $2 b$ contains approximately 11500 points, many fewer than our full oval radius dataset, and comparisons between $F_{\mathrm{PC}}$ and $\lambda$ are only possible for $\lambda<26^{\circ}$, otherwise the dayside portion of the oval encroaches into the dayglow and the OCB connot be identified. However, even this limited comparison shows a clear relationship between $\lambda$ and $F_{\mathrm{PC}}$. Superimposed on Fig. $2 \mathrm{~b}$ is the magnetic flux contained in a circle centred on $O$ as a function of radius $\lambda$ (full line). The polar cap is obviously smaller than our best-fit radius, so the dashed line shows the variation of $F_{\mathrm{PC}}$ as a function of oval radius, assuming that the OCB is $5^{\circ}$ of latitude poleward of the best-fit circle at all local times. This results in a good fit to the observations, not only giving us confidence that the oval radius is a proxy for the open flux content of the magnetosphere, but also allowing an extrapolation of the relationship between $\lambda$ and $F_{\mathrm{PC}}$ to larger oval radii. Hence, we find that oval radii of $15^{\circ}, 20^{\circ}$, $25^{\circ}$ and $30^{\circ}$ correspond approximately to open flux values of $0.25,0.5,1.0$, and $1.4 \mathrm{GWb}$.

For each FUV image we determine the concurrent value of the Sym-H index. We also employ observations from the Advanced Composition Explorer (ACE) spacecraft (Stone et al., 1998) to determine the upstream solar wind conditions. Solar wind velocity $\left(V_{\mathrm{SW}}\right)$, density $\left(N_{\mathrm{SW}}\right)$, and ram pressure $\left(P_{\mathrm{SW}}\right)$ were provided by the Solar Wind Electron, Proton and Alpha Monitor (SWEPAM) instrument (McComas et al., 1998) and the GSM components of the IMF $\left(B_{X}, B_{Y}\right.$, $B_{Z}$ ) by the MAG instrument (Smith et al., 1998). ACE observations were lagged to the magnetopause by considering the solar wind velocity. From these we estimate the dayside reconnection rate $\Phi_{D}$ as (Milan et al., 2008):

$\Phi_{D}=2.75 R_{E} V_{\mathrm{SW}} \sqrt{B_{Y}^{2}+B_{Z}^{2}} \sin ^{2} \frac{\theta}{2}$

where $\theta$ is the IMF clock angle and $R_{E}=6371 \mathrm{~km}$. The observations will be described in the next section.

\section{Observations}

We first discuss the variation of oval radius and Sym-H during three example intervals, each 11 days in duration, presented in Figs. 3, 4, and 5. In each figure we present oval radius $\lambda$, oval brightness $I_{\text {oval }}$, Sym-H, IMF $B_{Z}, V_{\mathrm{SW}}$ and $N_{\mathrm{SW}}$. Data-gaps in the two upper panels are caused by the orbit of the IMAGE spacecraft. Note the presence of sporadic patches of high oval radius data points, especially in Fig. 3; these are caused by the bad fits to the auroral oval described above. We now describe Fig. 3 in detail.

Three main enhancements in solar wind speed and solar wind density, which will each lead to a significant step in the solar wind dynamic pressure, are seen during this interval, marked by vertical dotted lines for clarity and labelled $A, B$, and $C$. $A$ and $C$ were followed by periods of strongly southward IMF, whereas $B$ was associated with mainly northward IMF. The impact on the magnetosphere of $A$ and $C$ is clearly apparent in Sym-H, which indicates the occurrence of two geomagnetic storms. The first storm $(A)$ had clear initial, main and recovery phases. Sym-H indicates that the storm re-intensified approximately a day into the main phase, associated with a period of strongly southward IMF. Initial, main, and recovery phases are also apparent in the second storm $(C)$. The second solar wind pressure enhancement $(B)$ produced a storm initial phase, but the subsequent negative excursion in Sym-H was very weak, and was delayed by $12 \mathrm{~h}$ 

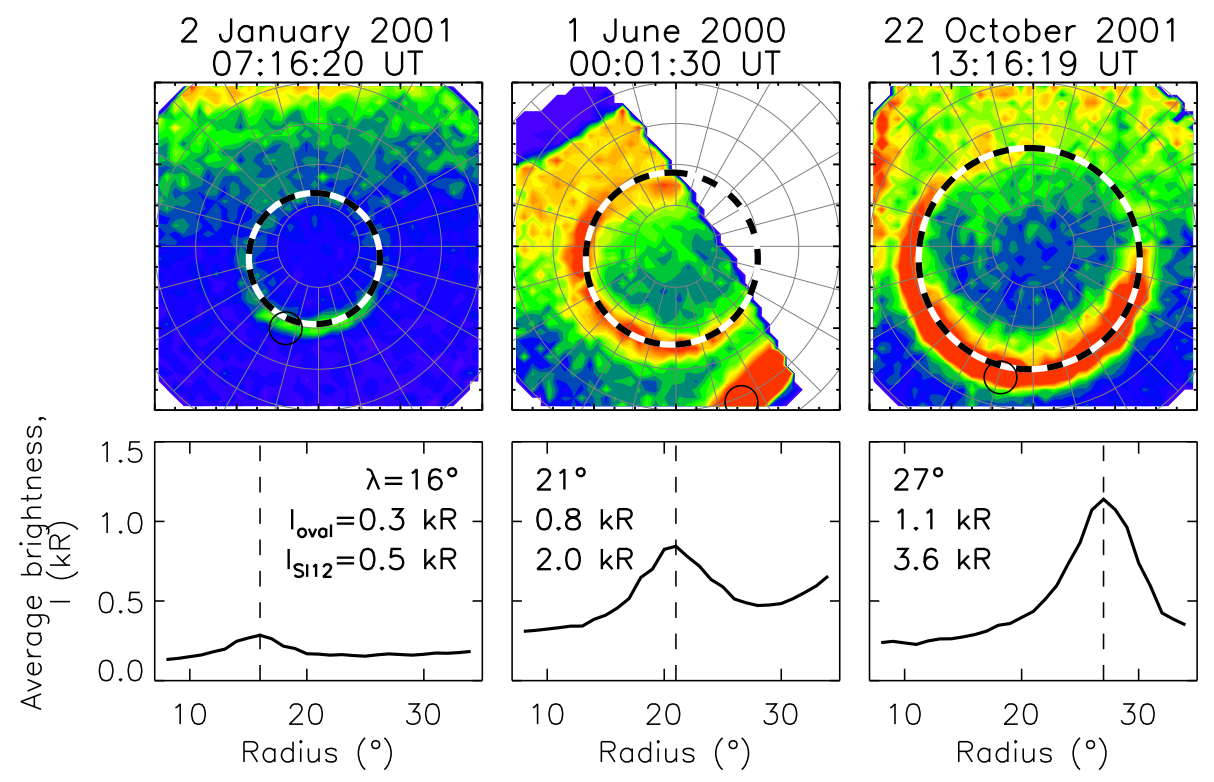

Fig. 1. Three example SI12 auroral images, demonstrating the fitting technique employed to determine the radius of the auroral oval. Lower panels show the variation of integrated brightness as a function of radius. The best-fit oval is shown by a black and white dashed circle in each panel. The peak of the nightside brightness is shown by small circles.

after the arrivel of the pressure step, only appearing once the IMF turned southward, albeit weakly. The upper two panels show that each pressure step was associated with an increase in the radius of the auroral oval, most significantly for $A$ and $C$, associated with the clearest storm signatures in Sym-H. In each case, the variation in oval radius closely mirrored the variation in Sym-H. In the case of $B$, the increase in oval radius was delayed until the southward turning of the IMF, again mirroring the behaviour of Sym-H. $A$ and $C$ were also associated with very significant enhancements in the SI12 auroral brightness, though the duration of these enhancements was limited to just a few hours after the solar wind step arrival.

Turning to Fig. 4, we highlight four events, $D, E, F$ and $G$. $D$ was associated with a sharp enhancement in solar wind density and a northward turning of the IMF. A prolonged initial phase was observed in Sym-H, there being a (weak) negative excursion only $18 \mathrm{~h}$ after the arrival of the pressure step when the IMF turned southwards. An enlargement in oval radius accompanied this step, but only once the IMF turned southwards. $E$ was associated with enhanced solar wind speed and a step in solar wind density. A clear initial phase was observed in Sym-H, followed $4 \mathrm{~h}$ later by a deep main phase once the IMF turned southwards. The oval radius was again elevated during this interval, but again only after the southward turning of the IMF. A small step in solar wind density, associated with a southward turning of the IMF, event $F$, lead to a re-intensification of the storm and a re-enlargement of the oval radius. Finally, at the end of the interval, a step in solar wind density and a southwards turn- ing in the IMF, event $G$, lead to the beginnings of a storm main phase and an increase in oval radius.

In the final example, Fig. 5, geomagnetic activity was driven by prolonged periods of southward IMF rather than steps in solar wind pressure. Sym-H did not display the characteristic storm behaviour, but showed a weak negative perturbation throughout much of the interval. However, as in the previous examples, the variation in oval radius closely mirrored the changes in the Sym-H index.

Before moving on, we examine the occurrence of substorms during storm $A$ of Fig. 3. Figure 6 focuses on a twoday subset of Fig. 3, and includes $I_{\mathrm{WIC}}$ as well as $I_{\text {oval }}$ and the auroral electrojet indices AU and AL as well as Sym-H. Highlighted by vertical dotted lines are the onsets of the major substorms which occurred during this interval (for which auroral images are available). Associated with most of these was an increase in oval radius prior to onset, the substorm growth phase when the open magnetic flux content of the magnetosphere increases through dayside reconnection, and a decrease in oval radius afterwards as magnetotail reconnection recloses open flux during the substorm expansion and recovery phases. The decreases in oval radius were accompanied by enhancements in $I_{\mathrm{WIC}}$, indicating significant auroral brightenings on the nightside, and also by substorm bays in the AL index. These observations are highly reminiscent of those reported by Milan et al. (2008) (see their Fig. 2), indicating that our oval radius measurement is sensitive to the change in the open flux content of the magnetosphere which occurs during the substorm cycle. It also shows that although the oval radius varies with the substorm cycle, during 


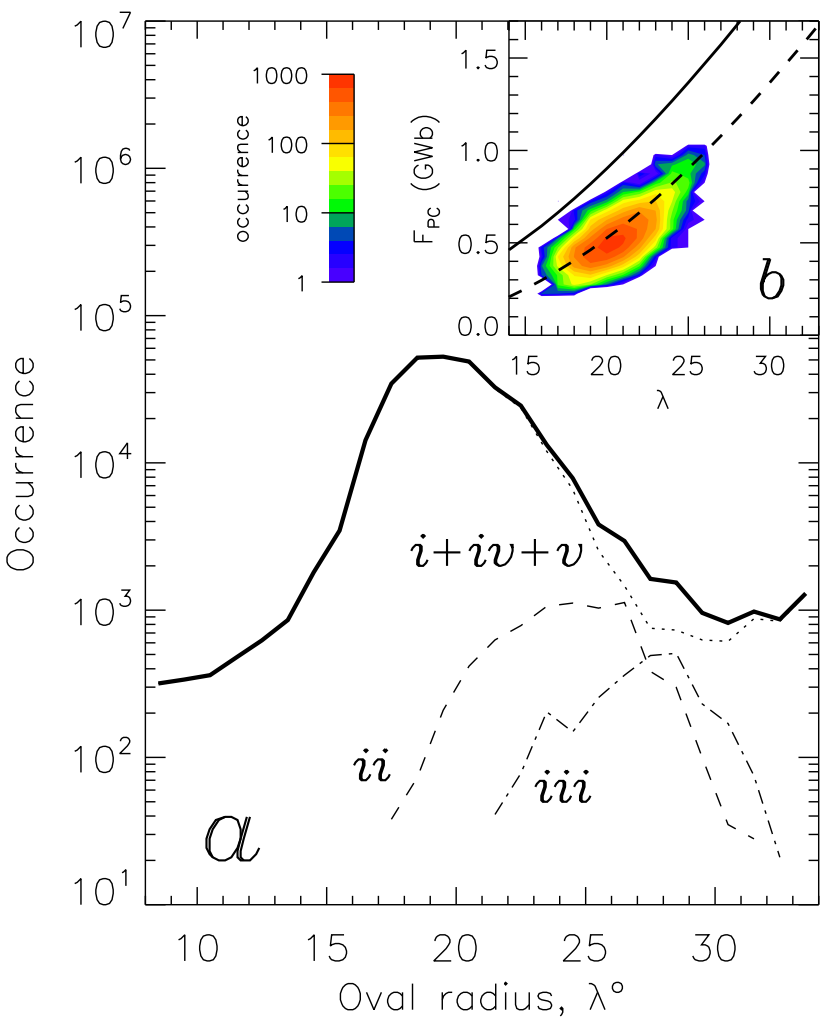

Fig. 2. (a) The distribution of oval radii for the two-year period June 2000 to May 2002. The solid curve shows the overall distribution. The distributions for Sym-H $>-75 n T,-75>$ Sym-H $>-125 n$ T, and Sym-H $<-125 \mathrm{nT}$ are also shown. (b) A comparison between oval radius $\lambda$ and open magnetic flux $F_{\mathrm{PC}}$, for a limited subset of the two-year period. Superimposed is the magnetic flux contained in a circle of radius $\lambda$ (full curve) and the open magnetic flux assuming that the OCB is located $5^{\circ}$ poleward of the circle of best-fit to the oval (dashed curve).

geomagnetic storms it remains considerably elevated above its quiet-time value.

As a final test of the substorm control of the radius of the auroral oval, we perform a superposed epoch analysis of oval radius keyed to substorm onset times identified by Frey et al. (2004), for the two-year period of our observations. The results are presented in Fig. 7. As described by Milan et al. (2009), the substorms are separated into five categories (IV) by their onset latitude, as reported by Frey et al. (2004), grouped in bins of $2^{\circ}$ of latitude, from the highest-latitude substorms (I, above $\left.68^{\circ}\right)$ to the lowest $\left(\mathrm{V}\right.$, below $\left.62^{\circ}\right)$. The numbers in the upper panel of Fig. 7 indicate the number of substorms which contributed to each category. In all categories except the highest-latitude onsets (I) there is a clear substorm signature consisting of an increase in oval radius prior to onset, followed by a decrease during the expansion phase, as described above in relation to Fig. 6. Moreover, the size of the variation depends on category, the lowest-

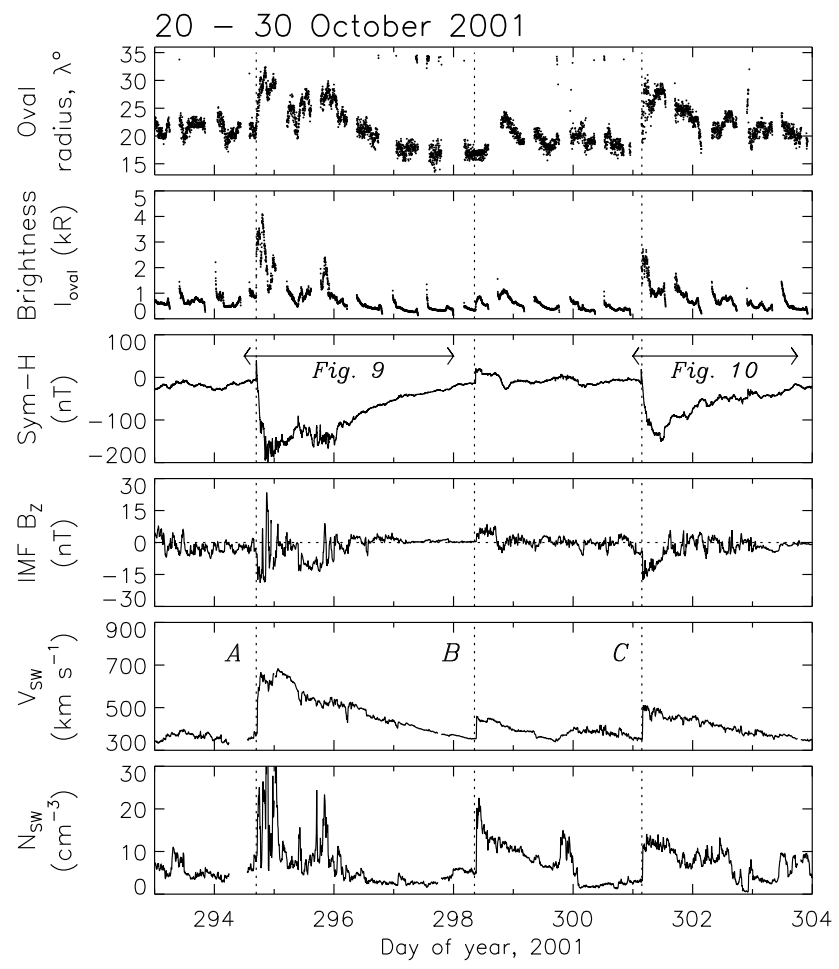

Fig. 3. The variation of oval radius $\lambda$, oval brightness $I_{\text {oval }}$, SymH, IMF $B_{Z}, V_{\mathrm{SW}}$ and $N_{\mathrm{SW}}$ for the 11-day interval 20-30 October 2001. Vertical dotted lines indicate solar wind features described in the text. Horizontal bars show the intervals studied in more detail in Figs. 8 and 9.

latitude substorms having the most significant change in oval radius. The lower panel of Fig. 7 presents a superposed epoch analysis of the IWIC measurements in the same categories as the upper panel, showing a clear enhancement in auroral brightness associated with the expansion phase, and a greater increase in brightness for lower-latitude substorms. These results are entirely consistent with the findings of Milan et al. (2009), who showed a greater variation in oval position and brightness for low latitude substorms, and Wild and Grocott (2008) and Grocott et al. (2009) who showed similar substorm-related variations in the size of the ionospheric convection pattern, as derived from SuperDARN observations.

Returning to Figs. 3, 4, and 5, we conclude that oval radius and the ring current intensity are closely related. We now examine the full two years of observations in a more statistical fashion. Figure 8 explores the dependence of the oval radius on the other available observations. For each available image we define the ordered pair $P=(\operatorname{Sym}-\mathrm{H}, \lambda)$. Figure $8 \mathrm{a}$ presents the occurrence distribution of points $P$ in bins of the oval radius of $1^{\circ}$ and bins of Sym-H of $10 \mathrm{nT}$, on a logarithmic colour scale. Figure $8 \mathrm{j}$ provides a key in which different areas within the parameter plane have been indicated to aid 


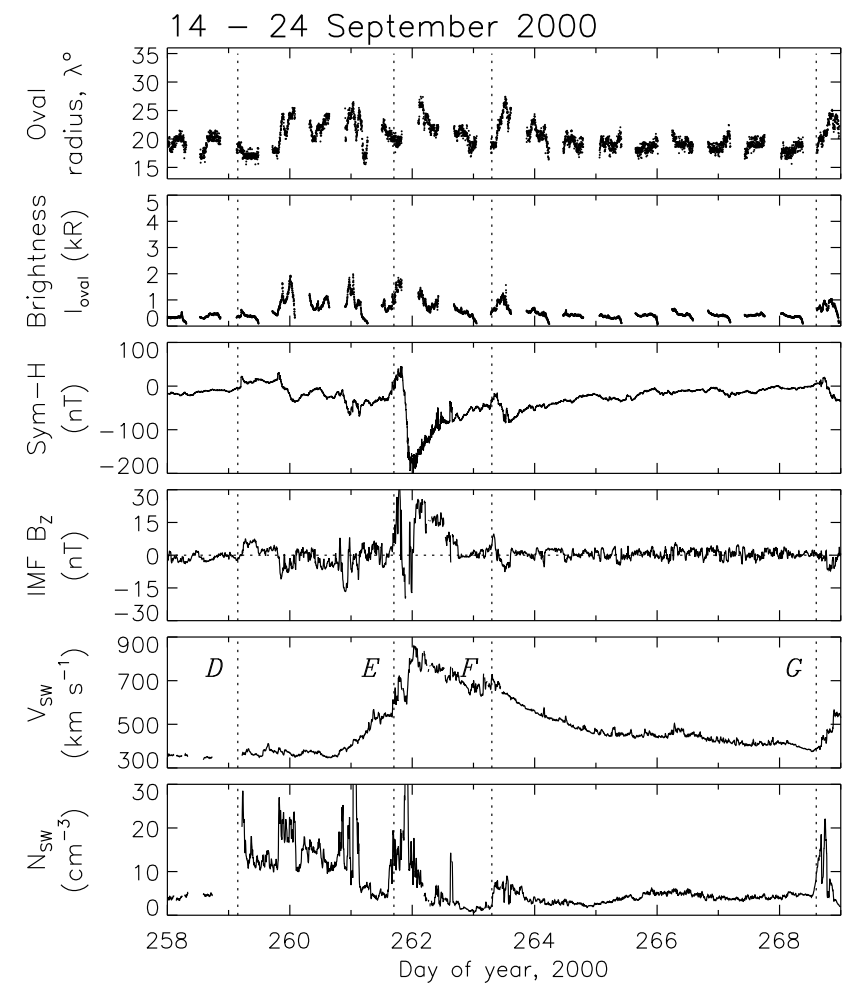

Fig. 4. Similar to Fig. 3 for the period 14-24 September 2000.

the following discussion. At this point we identify region $(v)$ which comprises bad fits occurring in images in which the auroral oval is very dim and the maximum in $I$ is associated with dayglow (large values of $\lambda$ ) or features in the background (lower values of $\lambda$ ). In the remaining panels of Fig. 8, cells with an occupancy greater than 10 are colourcoded by the average value in that cell of (b) $I_{\text {oval }}$, (c) $I_{\mathrm{SI} 12}$, (d) $I_{\mathrm{WIC}}$, (e) $V_{\mathrm{SW}}$, (f) IMF $B_{Z}$, (g) $N_{\mathrm{SW}}$, (h) $\Phi_{D}$, and (i) $P_{\mathrm{SW}}$. Most panels comprise data from approximately $240000 \mathrm{im}$ ages, less than in Fig. 2 due to gaps in availability of the ACE data. Figure $8 d$ contains fewer points as WIC images from summer months were discarded due to dayglow. The three main populations in Fig. 8 correspond to increasingly negative Sym-H, which we term (i) relatively undisturbed conditions, Sym-H $>-75 \mathrm{nT}$, (ii) moderate storm conditions, $-125 \mathrm{nT}>$ Sym-H $>-75 \mathrm{nT}$, and (iii) intense storm conditions, Sym- $\mathrm{H}<-125 \mathrm{nT}$. In addition, population $(i v)$, having mainly positive Sym-H, corresponds to storm initial phases.

Figure 8a supports our previous conclusion that there is a strong dependence of oval radius on Sym-H, the oval growing in radius as Sym- $\mathrm{H}$ becomes increasingly negative, varying from a mean value of approximately $18^{\circ}$ at $\mathrm{Sym}-\mathrm{H}$ of $0 \mathrm{nT}$ to close to $30^{\circ}$ as Sym-H dips to $-200 \mathrm{nT}$. This result is reiterated in Fig. 2, where the occurrence distribution of $\lambda$ has been subdivided by Sym-H into undisturbed conditions, a combination of populations $(i),(i v)$ and $(v)$, moderate storm conditons (ii), and intense storm conditions (iii).

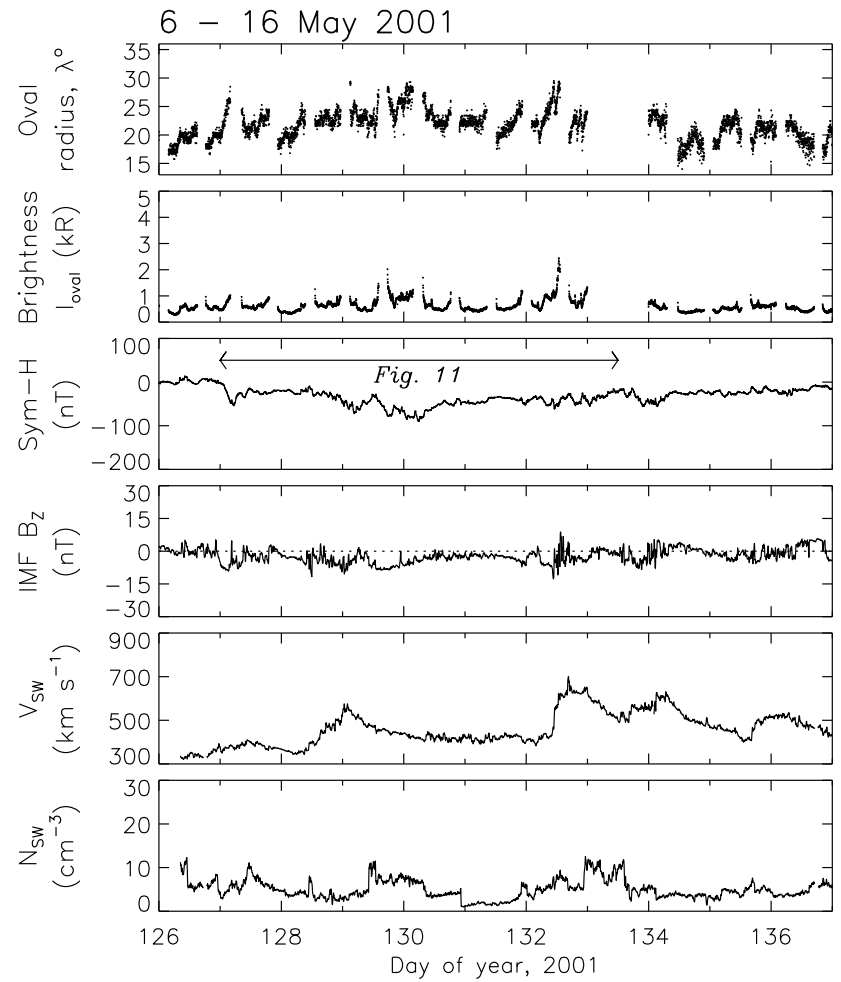

Fig. 5. Similar to Fig. 3 for the period 6-16 May 2001.

The SI12 overall brightness (Fig. 8b) and peak brightness (Fig. 8c) both increase with increasing oval radius, with almost no dependence on Sym- $\mathrm{H}$ except that brightness is also elevated for positive values of Sym-H corresponding to storm initial phases, population (iv). The WIC peak brightness (Fig. 8d) also increases with increasing oval radius, but is also greatest for large negative Sym-H, corresponding to population (iii). Proton aurora appear to respond strongly during storm initial phases, population (iv), but electrons in general do not.

We now examine each population of $P$ in turn. During quiet conditions $(i)$ we find low to moderate values of IMF $B_{Z}$ (averaging to near $0 \mathrm{nT}$ ), $\Phi_{D}, V_{S W}, N_{S W}$, and $P_{S W}$. It is clear, however, that within this population IMF $B_{Z}$ becomes more negative and $\Phi_{D}$ greater for increasing oval radius. There is also a tendence for $V_{S W}$ to increase towards moderate values for more negative Sym- $\mathrm{H}$.

During the initial phase of a storm, population (iv), we find high $N_{\mathrm{SW}}$ and $P_{S W}$, as expected. However, $V_{\mathrm{SW}}$ is not particularly elevated, on average. Within this population we find both positive and negative values of IMF $B_{Z}$, and corresponding low and high values of $\Phi_{D}$. Positive $B_{Z}$ is associated with large, positive Sym-H but low oval radii, whereas negative $B_{Z}$ is associated with larger oval radii, but less positively enhanced Sym-H. 

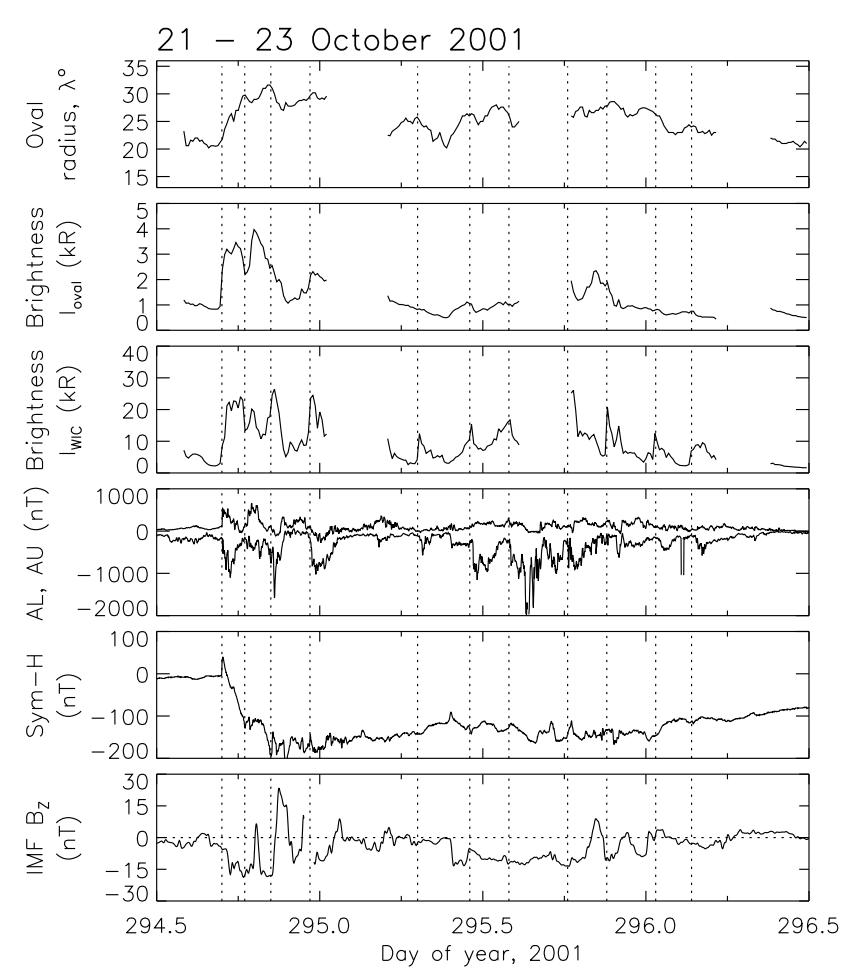

Fig. 6. A two-day subset of the time interval shown in Fig. 3, focusing on storm $A$, indicating the variation in oval radius $\lambda$, oval brightness $I_{\mathrm{Oval}}, I_{\mathrm{WIC}}, \mathrm{AU}$ and AL indices, Sym-H, and IMF $B_{Z}$.

During moderately disturbed periods $(i i)$, there is a clear dependence of oval radius on IMF $B_{Z}$ and $\Phi_{D}, B_{Z}$ being strongly positive at low oval radii and strongly negative at high oval radii. There also appears to be a positive correlation between oval radius and $N_{\mathrm{SW}}$ and, interestingly, a negative correlation between oval radius and $V_{\mathrm{SW}}$.

Finally, during intense storm conditions (iii), IMF $B_{Z}$ is strongly negative, $\Phi_{D}$ is enhanced, and $V_{\mathrm{SW}}$ is high. The $N_{\text {SW }}$ and $P_{\text {SW }}$ variations are more confused, though with a tendency towards higher densities at the largest oval radii.

On the whole we note that the $N_{\mathrm{SW}}$ and $P_{\mathrm{SW}}$ variations within the $P$ plane are very similar, and are markedly different from the variation of $V_{\mathrm{SW}}$. Although both $N_{\mathrm{SW}}$ and $V_{\mathrm{SW}}$ contribute to variations in $P_{\mathrm{SW}}$, the effect of varying $N_{\mathrm{SW}}$ dominates, for instance to produce storm inital phases. On the other hand, high $V_{\mathrm{SW}}$ is necessary to produce a deep main phase, through the contributions of $V_{\mathrm{SW}}$ and negative IMF $B_{Z}$ to a strong reconnection rate $\Phi_{D}$. For example, moderately disturbed conditions are possible for positive IMF $B_{Z}$ as long as $V_{\mathrm{SW}}$ is high, though oval radius remains low. Alternatively, moderate conditions can be attained for low $V_{\mathrm{SW}}$ as long as IMF $B_{Z}$ is strongly negative, in which case the oval radius is high. For very disturbed conditions, high $V_{S W}$ and strongly negative IMF $B_{Z}$ are necessary, and the oval radius is high.
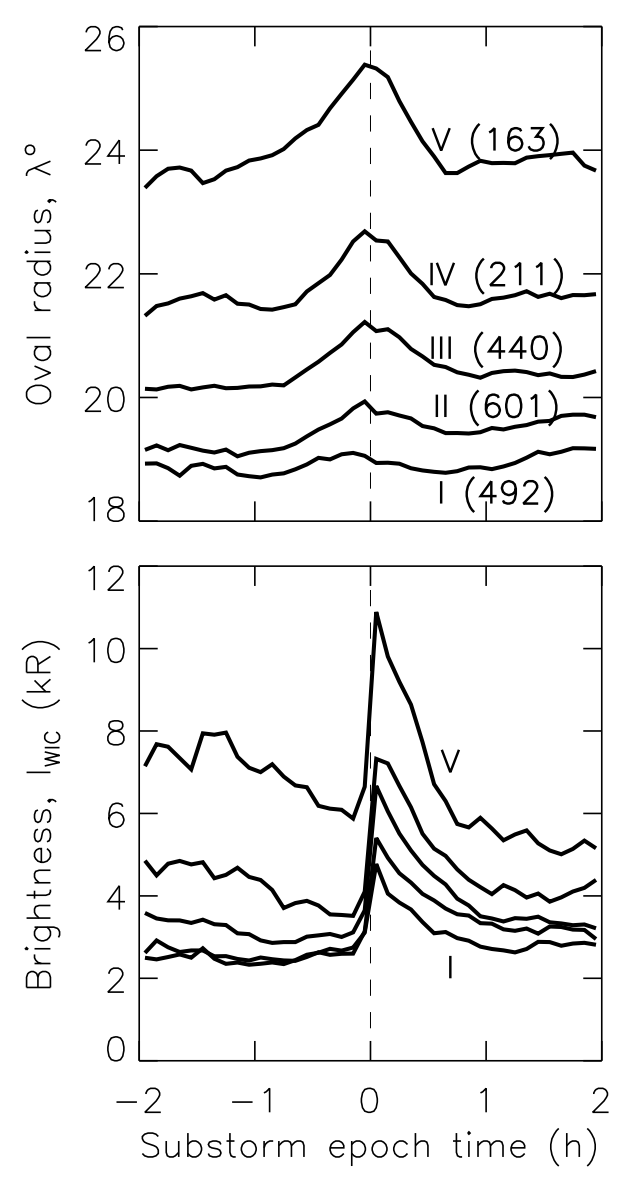

Fig. 7. A superposed epoch analysis of oval radius $\lambda$ and oval brightness $I_{\text {WIC }}$ keyed to substorm onset times reported by Frey et al. (2004). The substorms are separated into five categories, I to $\mathrm{V}$, depending on decreasing onset latitude (see text and Milan et al. (2009) for details). The numbers in brackets indicate the number of substorms within each category.

Finally, to help further interpret Fig. 8, we include three additional figures, Figs. 9, 10, and 11, presented in the same format, but for limited intervals of data previously studied in Figs. 3 and 5. Panel (j) of each figure shows the evolution of the point $P$ in time, colour coded from blue at the start of each interval to red at the end. These will be discussed in the next section.

\section{Discussion}

Our results indicate that the radius of the auroral oval increases for increasingly negative Sym-H (Fig. 8a). Such a relationship was previously suggested by Milan et al. (2008), who demonstrated an increase in magnetospheric open magnetic flux during geomagnetic storms. Specifically, in their Fig. 8 they demonstrated a decrease in the latitude of the onset arcs of substorms for more negative Sym-H, indicating 

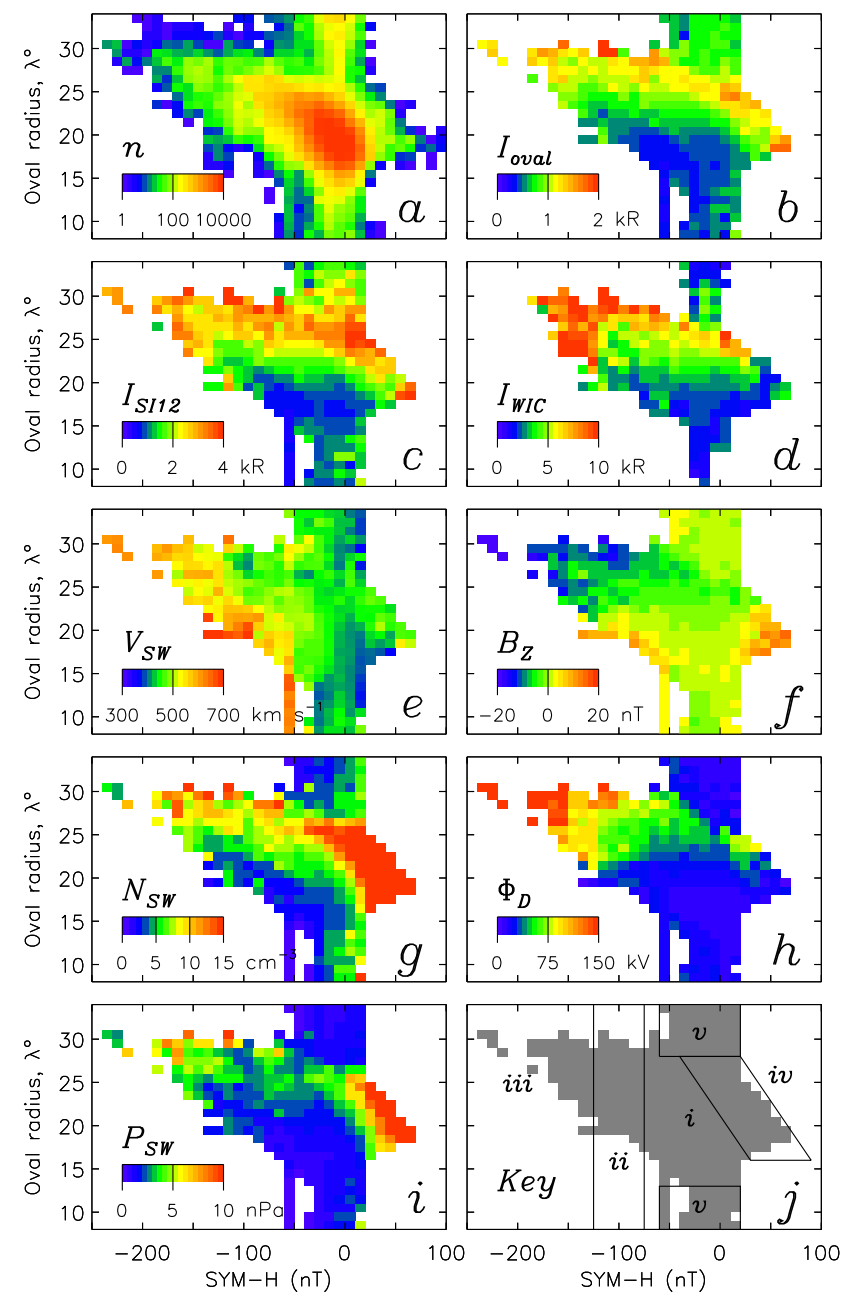

Fig. 8. A summary of oval radius $\lambda$ and Sym-H observations from the two-year period June 2000 to May 2002. (a) Occurrence distribution of the ordered pair $P=(\operatorname{Sym}-\mathrm{H}, \lambda)$. (b-i) Brightness and solar wind parameters binned and averaged within the $P$ plane: (b) overall SI12 brightness, $I_{\text {oval }}$; (c) peak SI12 brightness, $I_{\mathrm{SI} 12}$; (d) peak WIC brightness, $I_{\mathrm{WIC}}$; (e) solar wind velocity, $V_{\mathrm{SW}}$; (f) IMF $B_{Z}$; (g) solar wind density, $N_{\mathrm{SW}}$; (h) estimated dayside reconnection voltage, $\Phi_{D}$; (i) solar wind ram pressure, $P_{\mathrm{SW}}$. (j) A key indicating the regions $(i)$ to $(v)$ described in the text.

that substorms occur on an expanded auroral oval when the ring current is intensified. Yokoyama et al. (1998) also showed similar variations in the location of the equatorward edge of the auroral with $D_{s t}$. In addition, Milan et al. (2008) showed that the polar cap expands and contracts during the substorm cycle, as also supported by our Fig. 7, but during storm times remains enlarged above its quiet-time size; this is supported by Fig. 6 of the present paper. Finally, Milan et al. (2009) demonstrated that substorms occurring on expanded auroral ovals, i.e., those occurring during geomagnetic storms, are most intense and close the most open flux (see also Kamide et al., 1999); see also our Fig. 7.
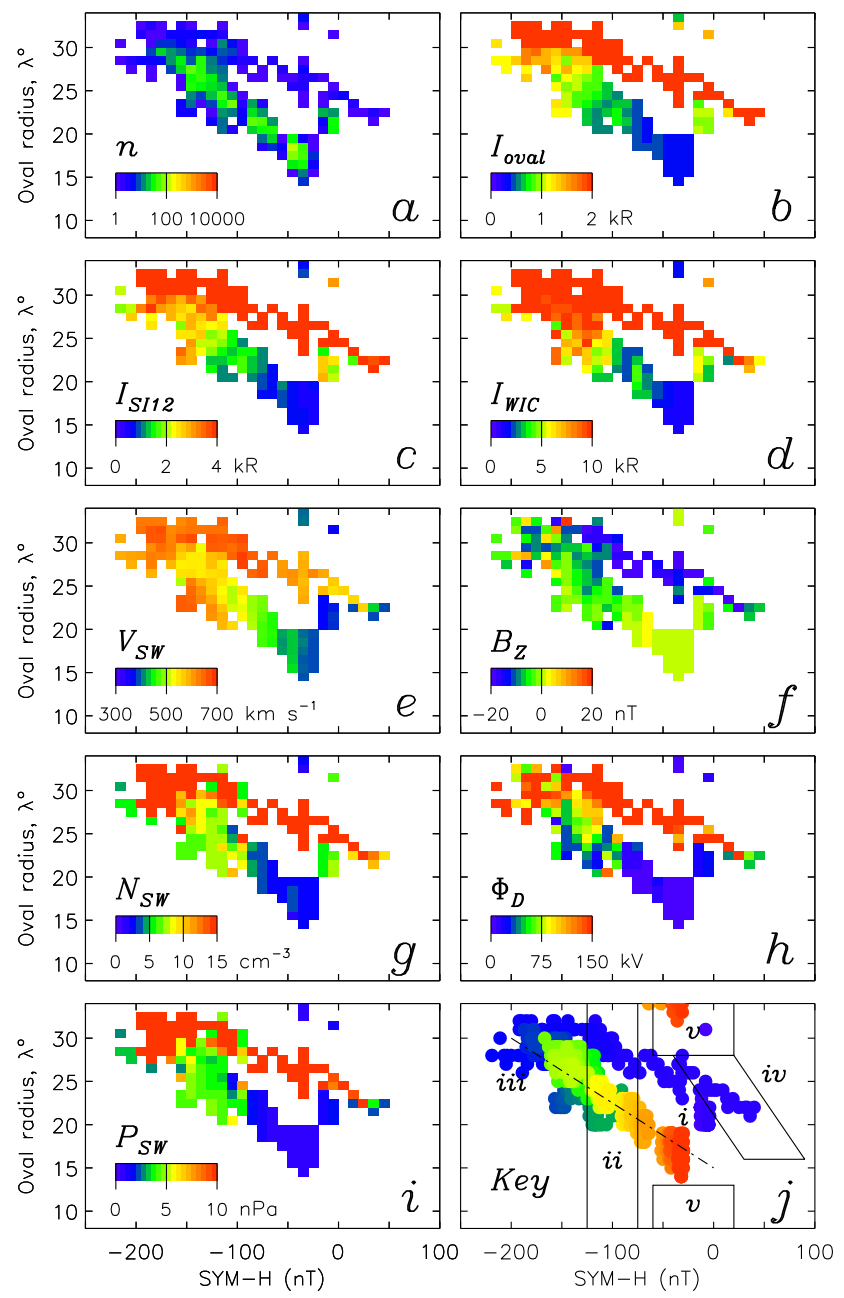

Fig. 9. Similar to Fig. 8, for the time interval indicated in Fig. 3. (j) The evolution of the position of point $P$, colour-coded from blue at the start of the interval to red at the end.

These observations are represented schematically in Fig. 12. Panel a shows the characteristic storm-time perturbation of Sym-H, indicating an enhanced ring current forming after the brief initial phase associated with the arrival of a pressure step in the solar wind. Panel $b$ then shows the typical behaviour of oval radius, which we assume is a proxy for polar cap size, that is the open flux content of the magnetosphere. Prior to the onset of the storm, the oval radius increases and decreases during substorm growth and expansion phases. The oval radius rises sharply at the onset of the storm, associated with intense dayside reconnection as the IMF turns strongly southward. Thereafter, the oval radius decreases gradually, mirroring the decay in Sym-H. However, during this decline the oval radius continues to expand and contract in response to continued southward IMF and the occurrence of substorms. Indeed, the substorm-associated fluctuations in oval radius are greater during the storm than 

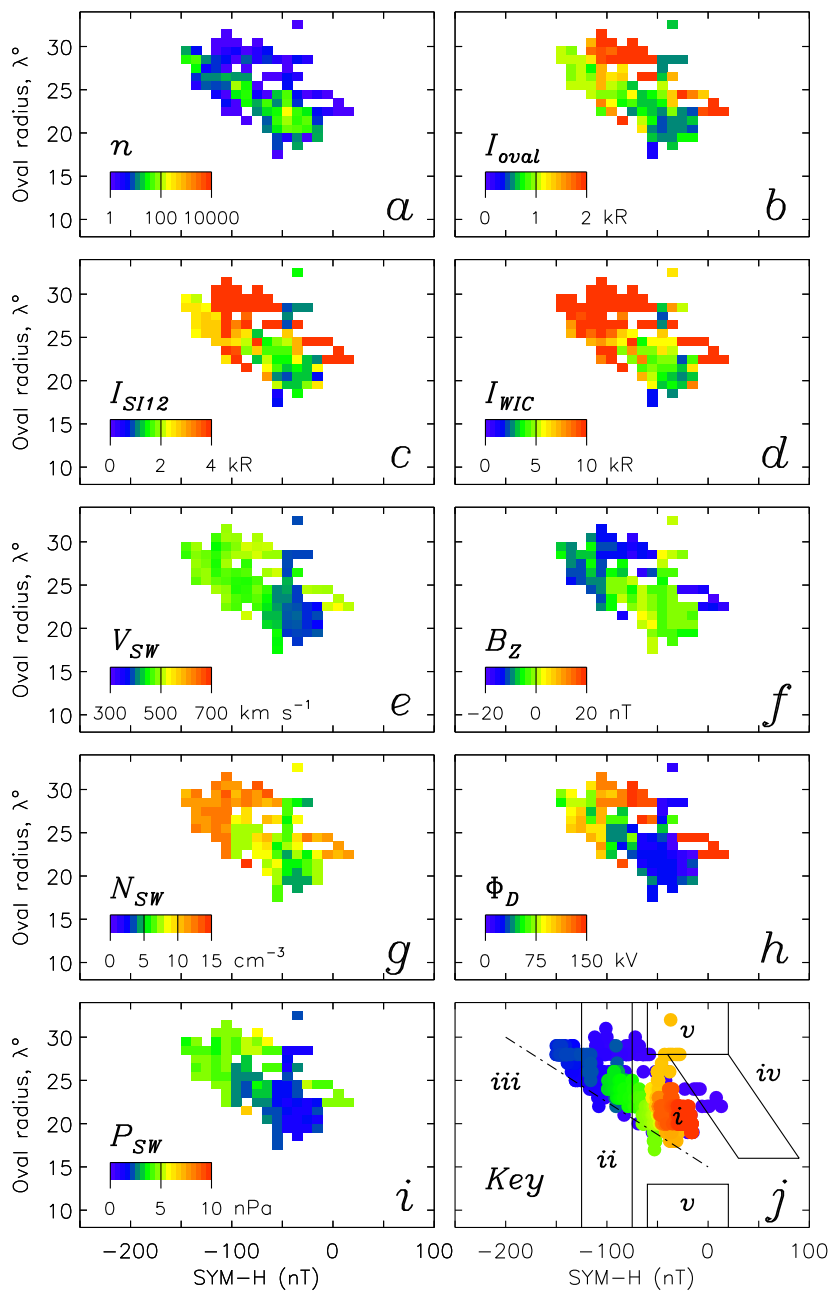

Fig. 10. Similar to Fig. 9, for the time interval indicated in Fig. 3.

before or after, as substorms occurring on an expanded oval have been shown to close more flux than substorms occurring during quieter conditions (Milan et al., 2009). We interpret these observations as indicating that there is an upper threshold in open flux above which reconnection is favoured in the magnetotail (dashed curve in Fig. 12b) and a lower threshold (dot-dashed curve) below which reconnection, if on-going, ceases. That is, if IMF $B_{Z}$ is negative, the oval radius increases until the upper threshold is reached, at which point tail reconnection is initiated and the oval radius decreases; once the lower threshold is attained tail reconnection ceases and the oval can expand again, if $B_{Z}$ continues to be negative. Moreover, these upper and lower reconnection thresholds are modulated by the intensity of the ring current.

As summarized by Slavin et al. (2002), substorms can be thought of as the magnetospheric response to increasing solar wind pressure on the magnetotail. As open flux is created by reconnection with the IMF at the dayside during substorm growth phase, the magnetotail becomes more flared, increas-
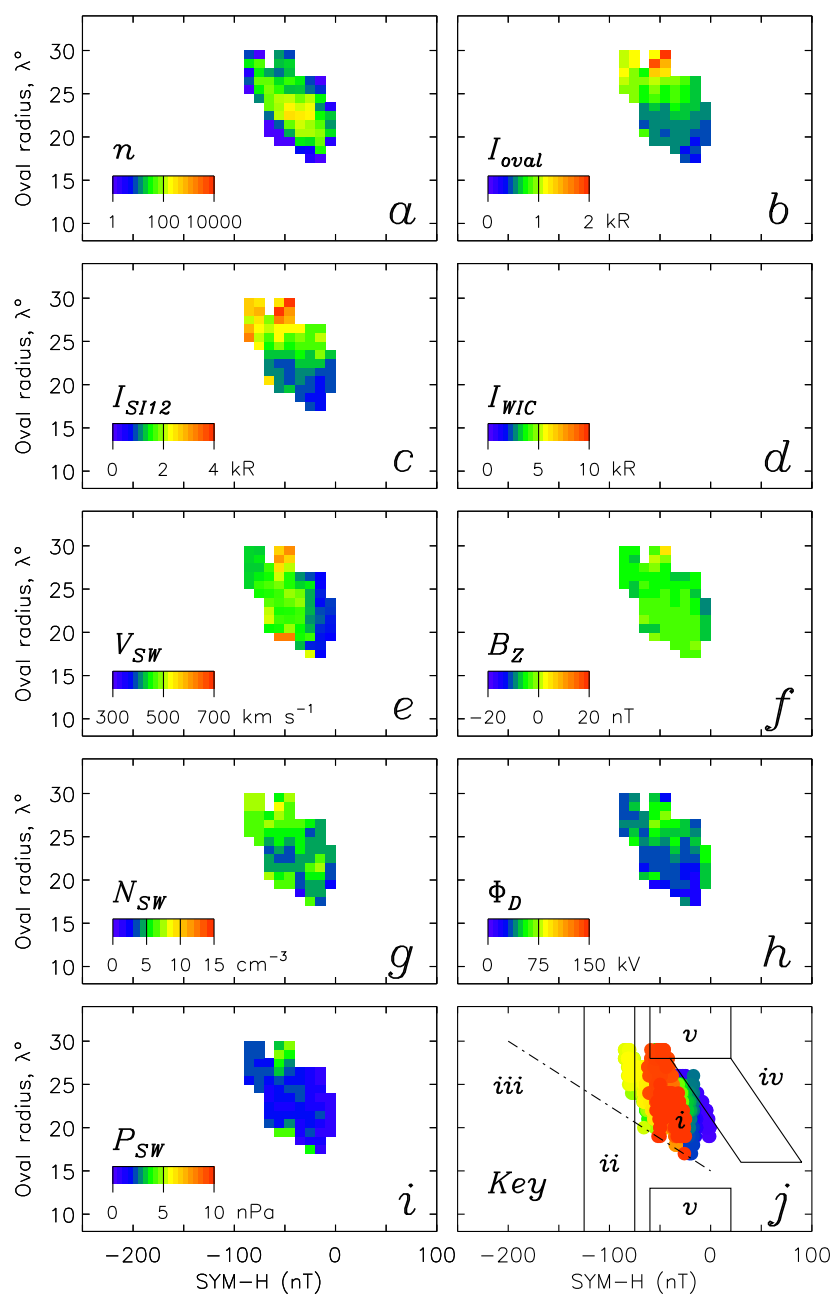

Fig. 11. Similar to Fig. 9, for the time interval indicated in Fig. 5.

ing the stress exerted by the solar wind on the tail magnetopause. This compresses the tail, leading to a stretched, taillike magnetic configuration and an intense cross-tail current. At some upper open flux threshold, the tail configuration becomes favourable for the onset of reconnection and substorm expansion occurs. Reconnection continues until the stress in the tail is relieved, that is when some lower open flux threshold is reached.

We interpret the increase in oval radius during storms as a stabilization of the magnetotail to the onset of reconnection and substorms by the magnetic perturbation associated with the westward-directed ring current (see also Nakai and Kamide, 2003; Milan et al., 2008, 2009). Inside the ring current region a negative $B_{Z}$ perturbation is produced, measured at the surface of the Earth as the negative Sym-H perturbation. Outside the ring current region a positive $B_{Z}$ perturbation dipolarizes the near-Earth tail, such that the onset of nightside reconnection is not favoured. In this situation the polar cap can expand to large values of open flux, moving 

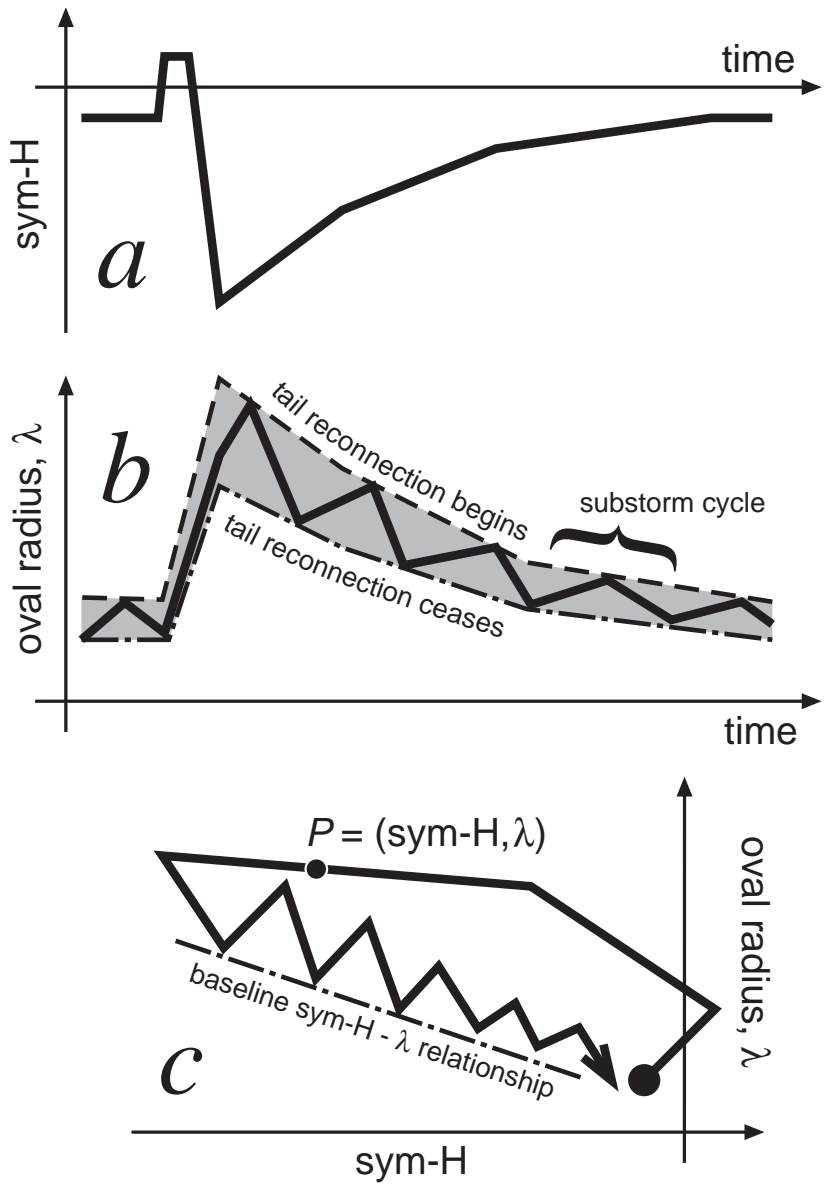

Fig. 12. A schematic indicating the expected variations of (a) Sym$\mathrm{H}$ and (b) oval radius $\lambda$ during the course of a geomagnetic storm. (c) The corresponding variation of the position of the ordered pair $P=($ Sym $-\mathrm{H}, \lambda)$ during the storm. See text for details.

the auroral oval to unusually low latitudes. It is only when the flaring angle of the magnetotail has grown to the point where the tail field is forced into a sufficiently stretched configuration that nightside reconnection and the occurrence of substorms are possible, that the polar cap can contract once again. In other words, the presence of the enhanced ring current increases the open flux threshold at which substorm onset is favoured. Under these conditions, contraction of the polar cap will cease while it is still enlarged, due to the continued dipolarizing effect of the ring current. That is, the lower open flux threshold is also increased. Hence, we interpret the increase in polar cap size during storms as a combination of increased coupling at the dayside, necessary for the polar cap to expand at all, but also as a modulation of the tail reconnection rate by the presence of an enhanced ring current. Enhanced dayside coupling, in itself, is not sufficient to explain the increase in oval radius during storms, as without the increase in the substorm threshold, tail reconnection would respond promptly and efficiently close open flux as quickly as it was produced.
Turning now to our other findings, Fig. 8b and c shows that the SI12 oval brightness is controlled mainly by the radius of the auroral oval, being greater when $\lambda$ is large. This variation can be understood in terms of pitch angle scattering of protons into the loss cone, as discussed previously by Blockx et al. (2005) and Milan et al. (2009): as the polar cap expands and the auroral oval moves to lower latitudes the nightside magnetic field becomes more stretched and pitch angle scattering of protons becomes efficient. The brightness of the proton aurora is also enhanced during the initial phases of storms, indicating that compressions of the magnetosphere also efficiently scatter protons into the loss cone. Electrons, with their smaller gyroradii, are not so efficiently scattered, either during storm initial phases or by large oval radii (Fig. 8d). Instead, precipitating electron fluxes are influenced more by substorm dynamics and a harder-driven magnetosphere, that is fore more negative Sym- $\mathrm{H}$, when IMF $B_{Z}$ is negative and $\Phi_{D}$ is large.

Our results suggest that IMF $B_{Z}$ and $V_{\mathrm{SW}}$ both play a role in determining the radius of the auroral oval and the stormtime ring current enhancement. Comparing Fig. 8e and f, we see that high solar wind speed and density and strongly southward IMF result in strong geomagnetic storm conditions with an expanded auroral oval. Moderate storm conditions with a contracted auroral oval are the result of high solar wind speed but predominantly northward IMF (resulting in low $\Phi_{D}$, see Fig. 8h); moderate storm conditions can also arise as a consequence of slower solar wind but strongly southward IMF (high $\Phi_{D}$ ), in which case the auroral oval has a large radius.

The relationship between $N_{\mathrm{SW}}$ and $P_{\mathrm{SW}}$ and the oval size is less clear. The initial phases of storms are associated with both high $N_{\mathrm{SW}}$ and high $P_{\mathrm{SW}}$, as expected. There then appears to be an anticorrelation between $V_{\mathrm{SW}}$ and $N_{\mathrm{SW}}$ during moderate storm conditions: low $V_{\mathrm{SW}}$ and high $N_{\mathrm{SW}}$ at large oval radii; high $V_{\mathrm{SW}}$ and low $N_{\mathrm{SW}}$ at low oval radii. As a consequence of this anticorrelation of $V_{\mathrm{SW}}$ and $N_{\mathrm{SW}}$, the behaviour of $P_{\mathrm{SW}}$, which is dependent on both $N_{\mathrm{SW}}$ and $V_{\mathrm{SW}}$, is somewhat confused in regions (ii) and (iii). The variation of oval radius with $V_{\mathrm{SW}}$ has been discussed above. The behaviour with $P_{\mathrm{SW}}$ is less clear, but perhaps can be understood in terms of the evolution of geomagnetic storms. That is, we should consider the evolution of the point $P$ represented by the ordered pair $P=(\mathrm{Sym}-\mathrm{H}, \lambda)$ as a storm progresses, as indicated schematically in Fig. 12c, and presented as data in Figs. 9, 10, and 11. We first concentrate on Fig. 9.

At the start of the interval a solar wind step (high $N_{\mathrm{SW}}$ and $\left.P_{\mathrm{SW}}\right)$ impinges on the magnetosphere and Sym-H becomes positive, $P$ moving from region $(i)$ into region $(i v)$. There is then a rapid intensification of the ring current and, especially as the IMF is strongly southward, a rapid expansion of the auroral oval to larger radii owing to the accumulation of new open flux though dayside reconnection; $P$ moves upwards and to the left (following the blue points in Fig. 9j), reaching region (iii) in the storm main phase. Conditions related to 
the storm onset, such as high solar wind density, high $\Phi_{D}$, and elevated SI12 brightnesses, are hence seen at high oval radii in regions $(i),(i i)$ and $(i i i)$. As the geomagnetic storm evolves through the recovery phase $P$ moves downwards and to the right (green to red points in Fig. 9j). This explains lower values of $N_{\mathrm{SW}}, \Phi_{D}$, and SI12 brightness at low oval radii in regions $(i)$ and $(i i)$. In other words, much of the behaviour in Fig. 8 can be understood in terms of a hysteresis in the motion of point $P$ during a storm, and the changing conditions through the storm. In a similar vein, Yokoyama et al. (1998) also noted that the latitude of the lower edge of the auroral oval tended to reach its maximum equatorward excursion somewhat before the minimum in the $D_{s t}$ main phase. Examination of Fig. 10 indicates a very similar behiaviour to Fig. 9.

During the recovery phase in both Figs. 9 and 10 there is an approximately linear relationship between Sym-H and $\lambda$, indicated by the dot-dashed line in panel (j):

$\lambda=15+0.075 \mid$ Sym-H $\mid$

This might be considered to be the base-line relationship between ring current intensity and oval radius. During the main phase, when solar wind coupling is particularly intense, the oval radius can be driven above this base-line curve; there will also be upward excursions relative to the base-line curve during the expansions and contractions of the polar cap related to the substorm cycle (see Fig. 12c).

In the third example, Fig. 11, there is no storm initial or well-defined main phase, as this storm is not associated with a step in solar wind density. In this case point $P$ moves to the upper left and back to the lower right, approximately following the base-line curve, with variations associated with the substorm cycle. Note that no WIC images were available during this interval.

\section{Conclusions}

The factors governing the size of the polar cap during storms and substorms are varied and complex. Strongly southward IMF is necessary for the polar cap to expand, such that the auroral oval moves to lower latitudes. Following steps in the solar wind pressure, periods of southward IMF are also responsible for intensifying the ring current to give the characteristic geomagnetic storm variation in Sym-H. The ring current then plays an important role in determining the threshold open flux at which substorm onset occurs, storm-time substorms occurring on an expanded auroral oval. Previous studies have indicated that substorms are more intense when they occur at low latitudes rather than at high latitudes. As the ring current is also known to be modulated by intense substorms and enhanced magnetospheric convection, it is interesting to speculate that this might constitute a feedback mechanism that keeps the ring current intensified when the magnetosphere is driven by enhanced coupling with the solar wind.

Acknowledgements. PDB was supported by a PPARC/STFC CASE award, grant no. PPA/S/C/2006/04488. The IMAGE FUV data were supplied by the NASA Space Science Data Centre (NSSDC), and we are grateful to the PI of FUV, S. B. Mende of the University of California at Berkeley, for its use. The ACE data used in this study were accessed through CDAWeb. The authors would like to thank N. F. Ness at the Bartol Research Institute and D. J. McComas of the Southwest Research Institute, for use of the MAG and SWEPAM data, respectively.

Topical Editor I. A. Daglis thanks C. Meng and another anonymous referee for their help in evaluating this paper.

\section{References}

Akasofu, S.-I. and Chapman, S.: The lower limit of latitude (US sector) of northern quiet auroral arcs, and its relation to $D_{s t}(H)$, J. Atmos. Terr. Phys., 25, 9-12, 1963.

Blockx, C., Gérard, J.-C., Meurant, M., Hubert, B., and Coumans, V.: Far ultraviolet remote sensing of the isotropy boundary and magnetotail stretching, J. Geophys. Res., 110, A11215, doi:10.1029/2005JA011103, 2005.

Boakes, P. D., Milan, S. E., Abel, G. A., Freeman, M. P., Chisham, G., Hubert, B., and Sotirelis, T.: On the use of IMAGE FUV for estimating the latitude of the open/closed magnetic field line boundary in the ionosphere, Ann. Geophys., 26, 2759-2769, 2008, http://www.ann-geophys.net/26/2759/2008/.

Boakes, P. D., Milan, S. E., Abel, G. A., Freeman, M. P., Chisham, G., and Hubert, B.: A statistical study of the open magnetic flux content of the magnetosphere at substorm onset, Geophys. Res. Lett., 36, L04105, doi:10.1029/2008GL037059, 2009.

Boudouridis, A., Zesta, E., Lyons, L. R., Anderson, P. C., and Lummerzheim, D.: Effect of solar wind pressure pulses on the size and strength of the auroral oval, J. Geophys. Res., 108, 8012, doi:10.1029/2002JA009373, 2003.

Burch, J. L.: IMAGE mission overview, Space Sci. Rev., 91, 1-14, 2000.

Burton, R. K., McPherron, R. L., and Russell, C. T.: An empirical relationship between interplanetary conditions and $D_{s t}$, J. Geophys. Res., 88, 4204-4214, 1975.

Caan, M. N., McPherron, R. L., and Russell, C. T.: Characteristics of the association between the interplanetary magnetic field and substorms, J. Geophys. Res., 82, 4837-4842, 1977.

Carbary, J. F., Sotirelis, T., Newell, P. T., and Meng, C.-I.: Auroral boundary correlations between UVI and DMSP, J. Geophys. Res., 108, 1018, doi:10.1029/2002JA009378, 2003.

Cliver, E. W.: The 1859 space weather event: Then and now, Adv. Space. Res., 38, 119-129, 2006.

Cowley, S. W. H. and Lockwood, M.: Excitation and decay of solar wind-driven flows in the magnetosphere-ionosphere system, Ann. Geophys., 10, 103-115, 1992.

Craven, J. D. and Frank, L. A.: Imaging results from Dynamics Explorer 1, Rev. Geophys., 26, 249-283, 1988.

Dungey, J. W.: Interplanetary magnetic fields and the auroral zones, Phys. Rev. Lett., 6, 47-48, 1961.

Feldstein, Y. I. and Starkov, G. V.: Dynamics of auroral belt and geomagnetic disturbances, Planet. Space Sci., 15, 209-229, 1967. 
Frey, H. U., Mende, S. B., Angelopoulos, V., and Donovan, E. F.: Substorm onset observations by IMAGE-FUV, J. Geophys. Res., 109, A10304, doi:10.1029/2004JA010607, 2004.

Grocott, A., Wild, J. A., Milan, S. E., and Yeoman, T. K.: Superposed epoch analysis of the ionospheric convection evolution during substorms: onset latitude dependence, Ann. Geophys., 27, 591-600, 2009, http://www.ann-geophys.net/27/591/2009/.

Hardy, D. A., Burke, W. J., Gussenhoven, M. S., Heineman, N., and Holeman, E.: DMSP/F2 electron observations of equatorward auroral boundaries and their relationship to the solar wind velocity and north-south component of the interplanetary magnetic field, J. Geophys. Res., 86, 9961-9974, 1981.

Holzworth, R. H. and Meng, C.-I.: Mathematical representation of the auroral oval, Geophys. Res. Lett., 2, 377-380, 1975.

Hubert, B., Milan, S. E., Grocott, G., Blockx, C., Cowley, S. W. H., and Gérard, J.-C.: Dayside and nightside reconnection rates inferred from IMAGE FUV and Super Dual Auroral Radar Network data, J. Geophys. Res., 111, A03217, doi:10.1029/2005JA011140, 2006.

Kamide, Y., Kokubun, S., Bargatze, L. F., and Frank, L. A.: The size of the polar cap as an indicator of substorm energy, Phys. Chem. Earth (C), 24, 119-127, 1999.

Lockwood, M. and Cowley, S. W. H.: Ionospheric convection and the substorm cycle, in: Proceedings of the International Conference on Substorms (ICS-1), 99-109, 1992.

McComas, D. J., Bame, S. J., Barker, P., Feldman, W. C., Phillips, J. L., Riley, P., and Griffee, J. W.: Solar Wind Electron Proton Alpha Monitor (SWEPAM) for the Advanced Composition Explorer, Space Sci. Rev., 86, 563-612, 1998.

Mende, S. B., Heetderks, H., Frey, H. U., Lampton, M., Geller, S. P., Habraken, S., Renotte, E., Jamar, C., Rochus, P., Spann, J., Fuselier, S. A., Gérard, J.-C., Gladstone, R., Murphree, S., and Cogger, L.: Far ultraviolet imaging from the IMAGE spacecraft. 1. System design, Space Sci. Rev., 91, 243-270, 2000a.

Mende, S. B., Heetderks, H., Frey, H. U., Lampton, M., Geller, S. P., Abiad, R., Siegmund, O. H. W., Tremsin, A. S., Spann, J., Dougani, H., Fuselier, S. A., Magoncelli, A. L., Bumala, M. B., Murphree, S., and Trondsen, T.: Far ultraviolet imaging from the IMAGE spacecraft. 2. Wideband FUV imaging, Space Sci. Rev., 91, 271-285, 2000b.

Meng, C.-I.: Latitudinal variation of the polar cusp during a geomagnetic storm, Geophys. Res. Lett., 9, 60-63, 1982.

Meng, C.-I.: Dynamic variation of the auroral oval during intense magnetic storms, J. Geophys. Res., 89, 227-235, 1984.

Meng, C.-I., Holzworth, R. H., and Akasfo, S.-I.: Auroral circle Delineating the poleward boundary of the quiet auroral belt, J. Geophys. Res., 82, 164-172, 1977.

Milan, S. E., Lester, M., Cowley, S. W. H., Oksavik, K., Brittnacher, M., Greenwald, R. A., Sofko, G., and Villain, J.-P.: Variations in the polar cap area during two substorm cycles, Ann. Geophys., 21, 1121-1140, 2003, http://www.ann-geophys.net/21/1121/2003/.

Milan, S. E.: Dayside and nightside contributions to the cross polar cap potential: placing an upper limit on a viscous-like interaction, Ann. Geophys., 22, 3771-3777, 2004, http://www.ann-geophys.net/22/3771/2004/.
Milan, S. E., Cowley, S. W. H., Lester, M., Wright, D. M., Slavin, J. A., Fillingim, M., Carlson, C. W., and Singer, H. J.: Response of the magnetotail to changes in open flux content of the magnetosphere, J. Geophys. Res., 109, A04220, doi:10.1029/2003JA010350, 2004.

Milan, S. E., Provan, G., and Hubert, B.: Magnetic flux transport in the Dungey cycle: A survey of dayside and nightside reconnection rates, J. Geophys. Res., 112, A01209, doi:10.1029/2006JA011642, 2007.

Milan, S. E., Boakes, P. D., and Hubert, B.: Response of the expanding/contracting polar cap to weak and strong solar wind driving: implications for substorm onset, J. Geophys. Res., 113, A09215, doi:10.1029/2008JA013340, 2008.

Milan, S. E., Grocott, A., Forsyth, C., Imber, S. M., Boakes, P. D., and Hubert, B.: A superposed epoch analysis of auroral evolution during substorm growth, onset and recovery: open magnetic flux control of substorm intensity, Ann. Geophys., 27, 659-668, 2009, http://www.ann-geophys.net/27/659/2009/.

Nakai, H., Kamide, Y., Hardy, D. A., and Gussenhoven, M. S.: Time scales of expansion and contraction of the auroral oval, J. Geophys. Res., 91, 4437-4450, 1986.

Nakai, H. and Kamide, Y.: Substorm-associated large-scale magnetic field changes in the magnetotail: a prerequisite for "magnetotail deflation" events, Ann. Geophys., 21, 869-879, 2003, http://www.ann-geophys.net/21/869/2003/.

Rostoker, G.: Triggering of expansive phase intensifications of magnetospheric substorms by northward turnings of the interplanetary magnetic field, J. Geophys. Res., 88, 6981-6993, 1983.

Russell, C. T., McPherron, R. L., and Burton, R. K.: On the cause of geomagnetic storms, J. Geophys. Res., 79, 1105-1109, 1974.

Shukhtina M. A., Dmitrieva, N. P., Popova, N. G., Sergeev, V. A., Yahnin, A. G., and Despirak, I. V.: Observational evidence of the loading-unloading substorm scheme, Geophys. Res. Lett., 32, L17107, doi:10.1029/2005GL023779, 2005.

Siscoe, G. L., and Huang, T. S.: Polar cap inflation and deflation, J. Geophys. Res., 90, 543-547, 1985.

Slavin, J. A., Fairfield, D. H., Lepping, R. P., Hesse, M., Ieda, A., Tanskanen, E., Østgaard, N., Mukai, T., Nagai, T., Singer, H. J., and Sutcliffe, P. R.: Simultaneous observations of earthward flow bursts and plasmoid ejection during magnetospheric substorms, J. Geophys. Res., 107, 1106, doi:10.1029/2000JA003501, 2002.

Smith, C. W., L'Heureux, J., Ness, N. F., Acuña, M. H., Burlaga, L. F., and Scheifele, J.: The ACE Magnetic Field Experiment, Space Sci. Rev., 86, 613-632, 1998.

Stone, E. C., Frandsen, A. M., Mewaldt, R. A., Christian, E. R., Marglies, D., Ormes, J. F., and Snow, F.: The Advanced Composition Explorer, Space Sci. Rev., 86, 1-22, 1998.

Wild, J. A. and Grocott, A.: The influence of magnetospheric substorms on SuperDARN radar backscatter, J. Geophys. Res., 113, A04308, doi:10.1029/2007JA012910, 2008.

Yokoyama, N., Kamide, Y., and Miyaoka, H.: The size of the auroral belt during magnetic storms, Ann. Geophys., 16, 566-573, 1998, http://www.ann-geophys.net/16/566/1998/. 